\title{
Analytical models for ellipticals and bulges with rotation
}

\author{
S.N. NURITDINOV \\ Tashkent University, Physical Faculty, Astronomy Department, Tashkent, Uzbekistan
}

\begin{abstract}
The results of the analytical study of two disspationless non-linear models are generalized in case of rotation.
\end{abstract}

\section{Non-Linear Models without Rotation}

The following non-linear models are very useful for the investigation of dissipationless collapse and the conditions of the formation of ellipticals: model 1 (see V.A. Antonov \& S.N. Nuritdinov, 1981, Sov.Astr.Zh., 58,1158)

$$
\Psi_{1}=\frac{\rho}{2 \pi v_{b}} \delta\left(v_{r}-v_{a}\right) \delta\left(v_{\perp}-v_{b}\right) \chi(\Pi-r)
$$

and model 2 (see S.N. Nuritdinov, 1983, Sov.Astr.Zh., 60, 40)

$$
\Psi_{2}=\frac{\rho \Pi^{3}}{\pi^{2}}\left[\left(\Pi^{2}-r^{2}\right)\left(\Pi^{-2}-v_{\perp}^{2}\right)-\Pi^{2}\left(v_{r}-v_{a}\right)^{2}\right]^{-\frac{1}{2}} \chi(\Pi-r) .
$$

Here $\rho(t)$ is the density at the time $t, v_{r}$ and $v_{\perp}$ are the radial and the transverse velocities, $\chi$ is the Heaviside step function, $\Pi=\frac{1+\lambda \cos (\psi)}{\left(1-\lambda^{2}\right)}, t=\frac{\psi+\lambda \sin (\psi)}{\left(1-\lambda^{2}\right)^{\frac{3}{2}}}, v_{a}=$ $\frac{-\lambda r \sin (\psi)}{\sqrt{1-\lambda^{2} \Pi^{2}}}, v_{b}=\frac{r}{\Pi^{2}}, 1-\lambda=\left(\frac{2 T}{|U|}\right)_{0}$. Antonov \& Nuritdinov $(1981)$ and Nuritdinov (1983) found the critical value $\Lambda_{\mathrm{cr}}=\frac{\sqrt{21}}{5}$ for the ellipsoidal oscillations mode that corresponds to the value of $\left(\frac{2 T}{|U|}\right)_{0}=0.084$ and that is connected with the radialorbit instability. The numerical experiments of L.Aguilar \& D.Merritt (1991, Ap.J., 345, 33) confirm this result. Nuritdinov (1985, Sov.Astr.Zh, 62, 506) noted an ellipsoidal instability zone $\lambda \in[0.611,0.873]$, which has a resonance nature ant corresponds to $\left(\frac{2 T}{|U|}\right)_{0} \in[0.127,0.389]$. Unfortunately up to now the accuracy of ous numerical experiments does not allow to reveal the presence of this zone.

\section{Models with Rotation}

For the case with rotation we have (see Nuritdinov, 1992, in press)

$$
\Psi_{\Omega}=\left[1+\Omega\left(\frac{v_{\perp}}{v_{b}}\right) \sin \theta \sin \gamma\right] \Psi_{1}, \quad \Psi_{\Omega}^{*}=\left(1+\Omega r v_{\perp} \sin \theta \sin \gamma\right) \Psi_{2}
$$

where $\sin \theta=\frac{\sqrt{x^{2}+y^{2}}}{r}, \tan \gamma=\frac{v_{\varphi}}{\theta}$, and $\Omega$ characterizes the rotation $(0 \leq \Omega \leq 1)$. The angular velocities of these models are $\omega_{1}=\frac{\Omega}{2 \Pi^{2}}$ and $\omega_{2}=\frac{\Omega}{4 \Pi^{2}}$. We found a critical dependence of $\left(\frac{2 T}{|U|}\right)_{0}$ on $\Omega$. It is interesting that the area of the radial-orbit instability connects to the resonance instability area at lowest value of $\Omega$. On the base of these models we can construct a new model $\Psi=(1-\nu) \Psi_{\Omega_{1}}+\nu \Psi_{\Omega_{2}}^{*}$, where $\nu, \Omega_{i}, i=1,2$ are free parameters. 


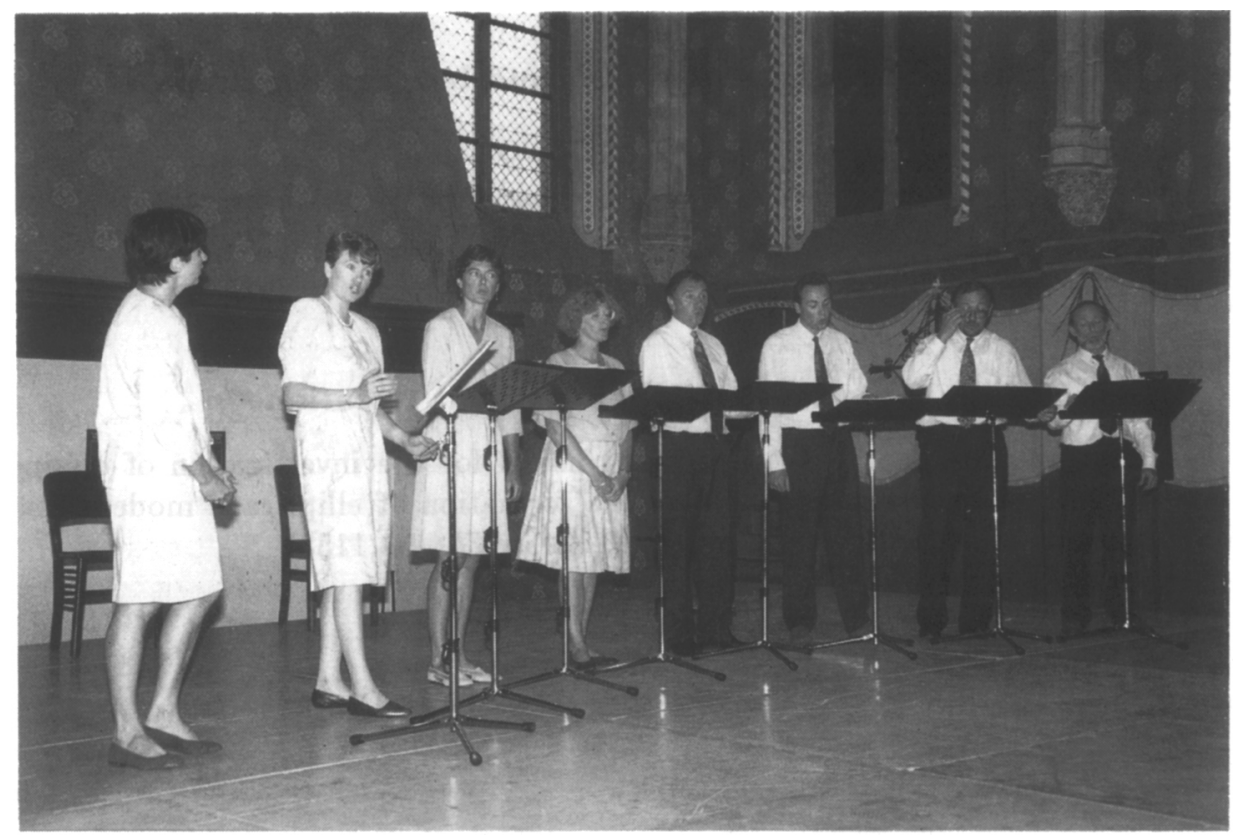

Singers and dancers performing during the cultural event

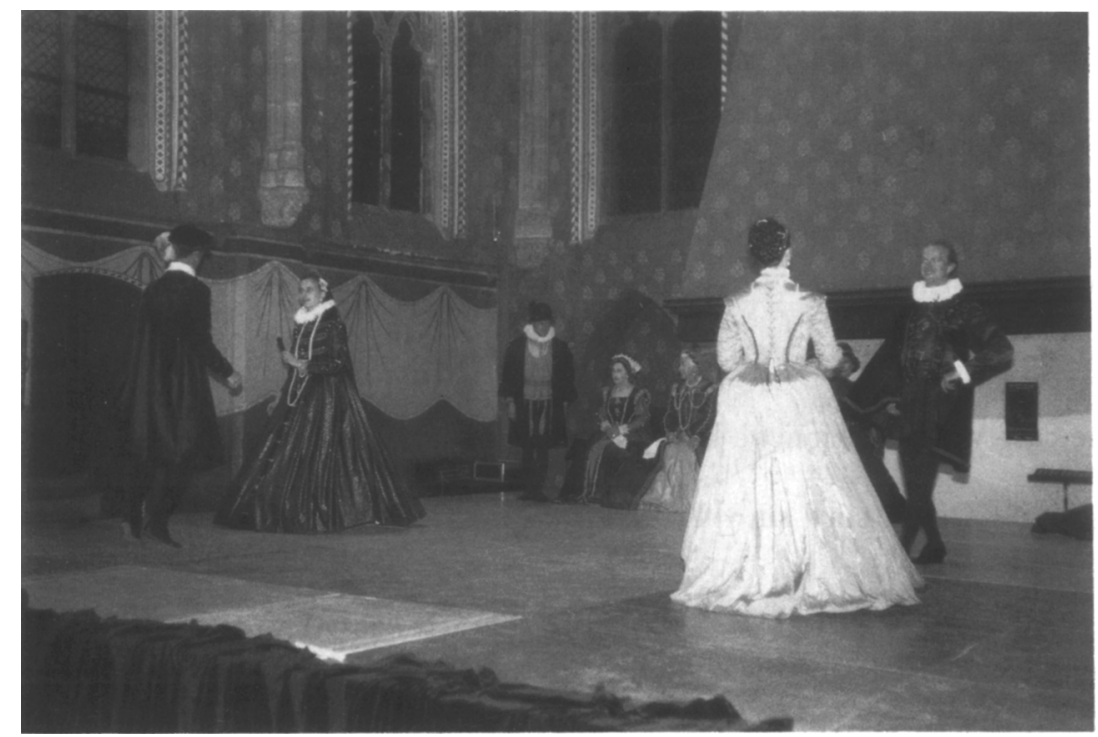

